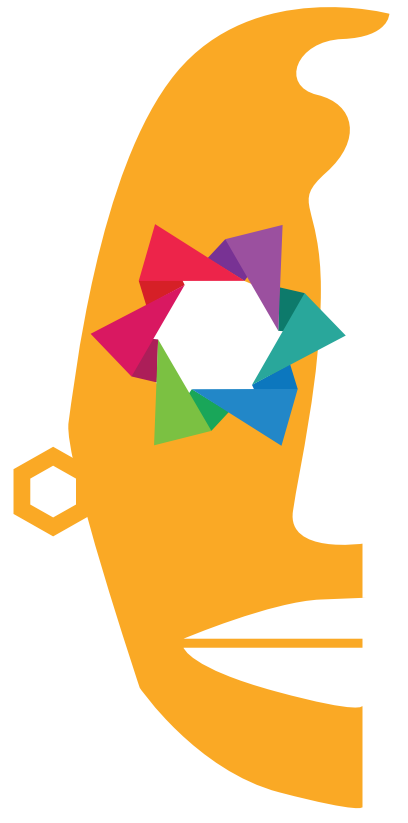

Leonardo

Candiano

Universidad de Buenos

Aires

Argentina

\title{
Los primeros dientes de El caimán barbudo $(1966-1967)^{1}$
}

\section{The first teeth of El caimán barbudo (1966-1967)}

\section{Resumen}

El artículo aborda las polémicas culturales desplegadas en Cuba en los inicios del magazine cultural de la Unión de Juventudes Comunistas, esencialmente las de El caimán barbudo, durante la permanencia de su primer equipo de redacción (1966-1967), que contó con la influencia notoria de la intelectualidad surgida a raíz del trabajo del Departamento de Filosofía de la Universidad de La Habana.

Para tal fin, se analizan los debates de su director, Jesús Díaz, publicados en las revistas Bohemia (1966) y La Gaceta de Cuba (1966), con Ana María Simó (antigua codirectora de la editorial El Puente) y con Jesús Orta Ruiz, conocido como El Indio Naborí. Asimismo, se retoma la polémica entre el equipo editorial de El caimán barbudo y el poeta Heberto Padilla en torno a las novelas de Lisandro Otero Pasión de Urbino (1966) y del ya por entonces emigrado Guillermo Cabrera Infante Tres tristes Tigres (1967).

De este modo, se inquiere en la heterogeneidad que distinguió la práctica cultural de la isla en el período, a la vez que se subraya el afán

1 Este artículo se enmarca en el Proyecto FiloCyT "Las representaciones del intelectual en disputa: el proceso cultural cubano entre 1959 y 1971 ", financiado por la Facultad de Filosofía y Letras de la Universidad de Buenos Aires y radicado en su Instituto de Filología y Literaturas Hispánicas. 
polemista que caracterizó las discusiones entre arte y política establecidas por las divergentes corrientes estéticas integradas al proceso revolucionario.

Palabras clave: Intelectualidad, Jesús Díaz, Revolución cubana, cultura, El caimán barbudo

\begin{abstract}
The present article approaches the cultural controversies developed in Cuba at the beginning of $E l$ caimán barbudo -the cultural magazine of the Union of Young Communists- especially during its first editing team, with evident influence of the intellectuality arisen from the work of the Philosophy Department of La Habana University.

For such purpose, this text analyses the debates of its director Jesús Díaz, published in the magazines Bohemia (1966) and La Gaceta de Cuba (1966), with Ana María Simó (co director of El Puente publishing house) and with Jesús Orta Ruiz, well known as El Indio Naborí. Besides, this work recovers the controversy between the editing team of El caimán barbudo and the poet Heberto Padilla surrounding the Lisandro Otero's novel Pasión de Urbino (1966) and Tres tristes Tigres (1967), a novel belonging to the already emigrated, in those times, Guillermo Cabrera Infante.

Therefore, this article inquires about heterogeneity that distinguished the cultural practice of the island in the period mentioned before, as well as highlights the confrotational eargerness that caracterized debates between art and politics established by the different aesthetic ideas integrated to the revolutionary process.
\end{abstract}

Keywords: Intellectuality, Jesús Díaz, Cuban Revolution, Culture, El caimán barbudo

\title{
Nace en La Habana un caimán con barba
}

La convivencia polémica entre posturas divergentes fue una constante en los primeros años del proceso revolucionario cubano, lo que motivó una prolífica cantidad de disputas públicas ampliamente reseñadas por la crítica especializada². Si el Proceso al sectarismo y el Gran Debate lo acreditan en lo político y en lo económico, la extendida querella entre "herejes" y "dogmáticos" de 1963 y 1964 lo plasma en el plano estético, del mismo modo que los desencuentros en torno del cortometraje $P M(1961)$ y las discusiones propuestas antes aún desde el magazine semanal Lunes de Revolución (1959-1961).

Esta heterogeneidad trascendió la conformación de espacios unitarios como la Unión Nacional de Escritores y Artistas de Cuba (UNEAC) en el ámbito cultural en 1961, y la fundación del Partido Comunista de Cuba (PCC) en octubre de 1965

2 Ver al respecto Graziella Pogolotti, ed., Polémicas Culturales de los sesenta (La Habana: Letras Cubanas, 2006); Jorge Fornet, El 71. Anatomía de una crisis (La Habana: Letras Cubanas, 2013); Ambrosio Fornet, $<<$ Quinquenio Gris: revisitando el término $>>$, Criterios, (2007); Julio César Guanche, El continente de lo posible (La Habana: Ruth Editorial, 2008); Claudia Gilman, Entre la pluma y el fusil (Siglo XXI: Buenos Aires, 2003); Néstor Kohan, $<<$ Pensamiento Crítico y el debate sobre las Ciencias Sociales en el seno de la Revolución Cubana>>, en Crítica y teoría en el pensamiento social latinoamericano (CLACSO: Buenos Aires, 2006), 389-435; entre otros. 
en lo político-institucional. El surgimiento de El caimán barbudo, en marzo de 1966, fue una de las vías por las que esta diversidad se expresó durante el segundo lustro de los años '60. Este trabajo pretende comprobar esta afirmación a partir de un análisis de los posicionamientos que sus principales referentes desplegaron contra otras corrientes de pensamiento integradas a la Revolución.

El caimán es una publicación cultural fundada como suplemento de Juventud Rebelde, órgano oficial de la Unión de Juventudes Comunistas -UJC- desde 1965. En sus comienzos era gratuito y su tiraje mensual rondaba los 80.000 ejemplares, lo que lo convirtió en uno de los medios culturales más masivos de la historia de la isla ${ }^{3}$. Su primer director fue el escritor y profesor Jesús Díaz, que en enero del ' 66 había recibido el premio continental Casa de las Américas por su volumen de cuentos Los años duros (1966). Este autor logró absoluta autonomía para organizar el mensuario, reunir un equipo de trabajo y publicar los artículos y textos que consideró pertinentes, decisión que no llama la atención debido a que Díaz pertenecía al Departamento de Filosofía de la Universidad de La Habana, equipo intelectual ideológicamente cercano al liderazgo revolucionario, tal como lo acredita Martínez Pérez en su trabajo Los hijos de Saturno (2006).

Figura 1. Portada del número de El caimán barbudo del mes de junio de 1966

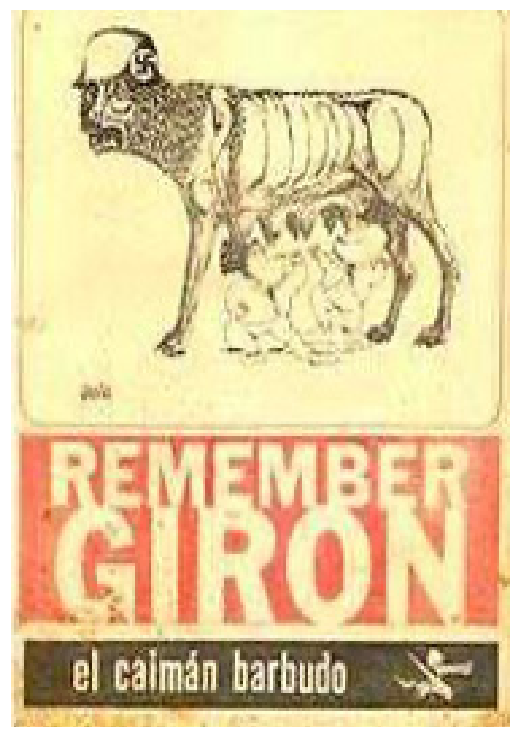

Fuente: https://www.ecured.cu/El_Caim\%C3\%A1n_Barbudo

3 Solo superada por Lunes de Revolución, que entre 1959 y 1961 llegó a tirar 250.000 ejemplares. Ver Leandro Estupiñan, Lunes: un día de la Revolución Cubana (Buenos Aires: Dunken, 2015). 
En el marco de este vertiginoso proceso político iniciado en 1959, la aparición de El caimán barbudo respondió a su específica coyuntura histórica, derivada en parte de la conformación del Partido Comunista de Cuba y, como señalan Guerra Vilaboy y Maldonado en su Historia de la Revolución Cubana ${ }^{4}$, del paulatino distanciamiento de la isla respecto de ciertos lineamientos de la política soviética.

Resultan reconocidas en la actualidad las múltiples discrepancias de Cuba para con la URSS durante el desarrollo de los años '60. Rafael Hernández afirma que Cuba en este período afrontó: "El desafío de construir un socialismo distinto y distante de los modelos soviético y chino"s, y que no eran un secreto las divergencias entre la línea del socialismo cubano por un lado, y la de las potencias socialistas por el otro. Julio César Guanche precisa aún más el momento de esta demarcación en el interior del campo anticapitalista al establecer que: "El lapso 1966-1967 marcaría el cenit de la política independiente del socialismo cubano, de la cual fue punta de lanza la promoción de la lucha armada en Latinoamérica, en franca contradicción con los intereses soviéticos en la región", hecho en el que remarca el protagonismo de los miembros del Departamento de Filosofía. Del mismo modo, Ambrosio Fornet rememora que en estos años: "se puso de manifiesto que gran parte de nuestra intelectualidad estaba elaborando, desde posiciones martianas y marxistas, un pensamiento descolonizador, más ligado a nuestra realidad y a los problemas del Tercer Mundo"”.

Efectivamente, la fundación del PCC dio espacio preponderante al antidogmatismo, hecho que se verificó en los siguientes 3 años con el intento de constitución de un nuevo andamiaje teórico e intelectual que tuvo en los integrantes del Departamento de Filosofía a parte de sus principales referentes. Por ello Martínez Pérez sostiene que las características de la práctica cultural de El caimán estuvieron modeladas por el contexto revolucionario del que emergió y en el cual se desarrolló, estableciéndose una correlación entre las interpretaciones políticas y estéticas de sus integrantes con la acción y el discurso hegemónicos en esos años en la isla, un período determinado por la construcción de un camino propio para el socialismo cubano y por una débil e incipiente institucionalidad que explica la autonomía de la dirección del mensuario respecto de las normativas de las organizaciones político-culturales a las que oficialmente debía responder, fundamentalmente la UJC.

4 Ver Sergio Guerra Vilaboy, Alejo Maldonado, Historia de la Revolución Cubana (Txtalaparta: Navarra, 2009).

5 Rafael Hernández, <<El año rojo. Política, sociedad y cultura en 1968〉>, Revista de Estudios Sociales $\mathrm{N}^{\circ}$ 33 (2009): 44.

6 Julio César Guanche, El continente de lo posible..., 62.

7 Ambrosio Fornet, <<Quinquenio Gris... >>, 11. 
De este modo, la primera época de El caimán fue un producto de su tiempo, y tanto por su masividad como por su acercamiento a los posicionamientos del liderazgo político resulta imprescindible de analizar para comprender el itinerario histórico de la Revolución cubana, mucho más si consideramos que desde sus orígenes trascendió los márgenes de una publicación, pues bajo su promoción se destacó la continua organización de conciertos musicales, galerías de arte, recitales de poesía, mesas debate y concursos a través de los que emergió un movimiento artístico juvenil.

Díaz se mantuvo al frente del proyecto hasta noviembre de 1967, cuando tanto él como la totalidad del Consejo de Redacción fueron reemplazados, lo que provocó el abrupto final de lo que se conoció como la "primera época" de El caimán. Durante este lapso, el magazine contó, entre sus principales integrantes, con Guillermo Rodríguez Rivera, Luis Rogelio Nogueras, Orlando Alomá, Víctor Casaus y Ricardo Machado, a los que se sumaron como asiduos colaboradores Hugo Azcuy, Aurelio Alonso y Fernando Martínez Heredia. Estos últimos tres, al igual que Machado, pertenecientes también al Departamento de Filosofía. El rango etario de los participantes expresó la irrupción de una nueva camada al frente de uno de los más importantes órganos de difusión de la isla: Jesús Díaz tenía por entonces 24 años y era de los más veteranos, pues superaba a Rodríguez Rivera, Alomá y Casaus, todos con 23, y a Nogueras, de 22.

El lugar otorgado a estos intelectuales, los primeros formados en su educación superior íntegramente por la Revolución, asumió el carácter de apuesta. Si en "El socialismo y el hombre en Cuba" (1965) el Che había cuestionado, por un lado, a los ortodoxos debido a su dogmatismo y, por el otro, a los herejes por no ser verdaderamente revolucionarios, también había afirmado: “ya vendrán los revolucionarios que entonen el canto del hombre nuevo con la auténtica voz del pueblo"8. Rápidamente, los integrantes de El caimán se autoproclamaron como tales en su editorial inaugural: "comprometidos con la Revolución y con el arte hasta la médula de los huesos, entonaremos desde estas páginas el canto nuevo, alegre y triste, esperanzado y cierto de los constructores" $"$. Parecía que ese futuro anhelado por Guevara se concretaba con suma celeridad.

Quizás por ello a poco del nacimiento de esta publicación su máximo referente inició disputas contra diversos intelectuales igualmente integrados al proceso revolucionario, con lo que le estableció continuidad al carácter polémico de la dinámica intelectual de la isla. Para ello aprovechó la invitación a participar de dos encuestas, una del número de abril-mayo de 1966 de La Gaceta de Cuba que se

8 Ernesto Guevara, <<El socialismo y el hombre en Cuba〉>, en Lecturas para la reflexión $N^{\circ}$ 3. Economía y hombre nuevo (China: Ocean Press, 2013), 86.

$9<<$ Editorial $>>$, El caimán barbudo $\mathrm{N}^{\circ} 1,(1966)$ : 1. 
centró en las miradas sobre la perspectiva generacional dentro de la Revolución, y otra que reunió a doce intelectuales para discutir sobre literatura revolucionaria y que fue publicada por Bohemia el 22 de julio del mismo año.

\section{Por una vanguardia popular}

La encuesta de Bohemia fue realizada a Alejo Carpentier, José Antonio Portuondo, Nicolás Guillén, Jaime Sarusky, Edmundo Desnoes, Heberto Padilla, Roberto Fernández Retamar, Alfredo Guevara, Pablo Armando Fernández, Ambrosio Fornet, Lisandro Otero y Jesús Díaz, quienes respondieron respecto del vínculo entre literatura y revolución, el lugar del realismo en la representación estética del proceso cubano y las principales obras del período. Asimismo, efectuaron un balance de la producción literaria de los últimos años en la isla. Como se verifica con la simple enumeración de los participantes, se pretendió difundir el pensamiento de los por entonces responsables primordiales de la literatura cubana; ya que la mayoría de ellos, además de llevar adelante una obra propia, dirigían las más significativas instituciones y publicaciones culturales del país. Carpentier había sido hasta poco tiempo atrás director de la Editorial Nacional y comenzaba sus labores diplomáticas como embajador en Francia, Guillén presidía la UNEAC, Portuondo era rector de la Universidad de Oriente, Retamar dirigía la revista Casa de las Américas, Otero era el vicepresidente del Consejo Nacional de Cultura (CNC) y Alfredo Guevara el director del ICAIC. Sarusky, Pablo A. Fernández, Desnoes y Fornet detentaban responsabilidades en la gestión de la cultura en diversos ámbitos y colaboraban de manera cotidiana en medios gráficos de la isla al igual que Padilla, de reciente regreso de la URSS. La inclusión de Díaz en este grupo evidencia el espacio otorgado a los jóvenes de El caimán apenas meses después de iniciadas sus labores culturales. 
Figura 2. Respuesta de Díaz sobre "Literatura Revolucionaria" en revista Bohemia

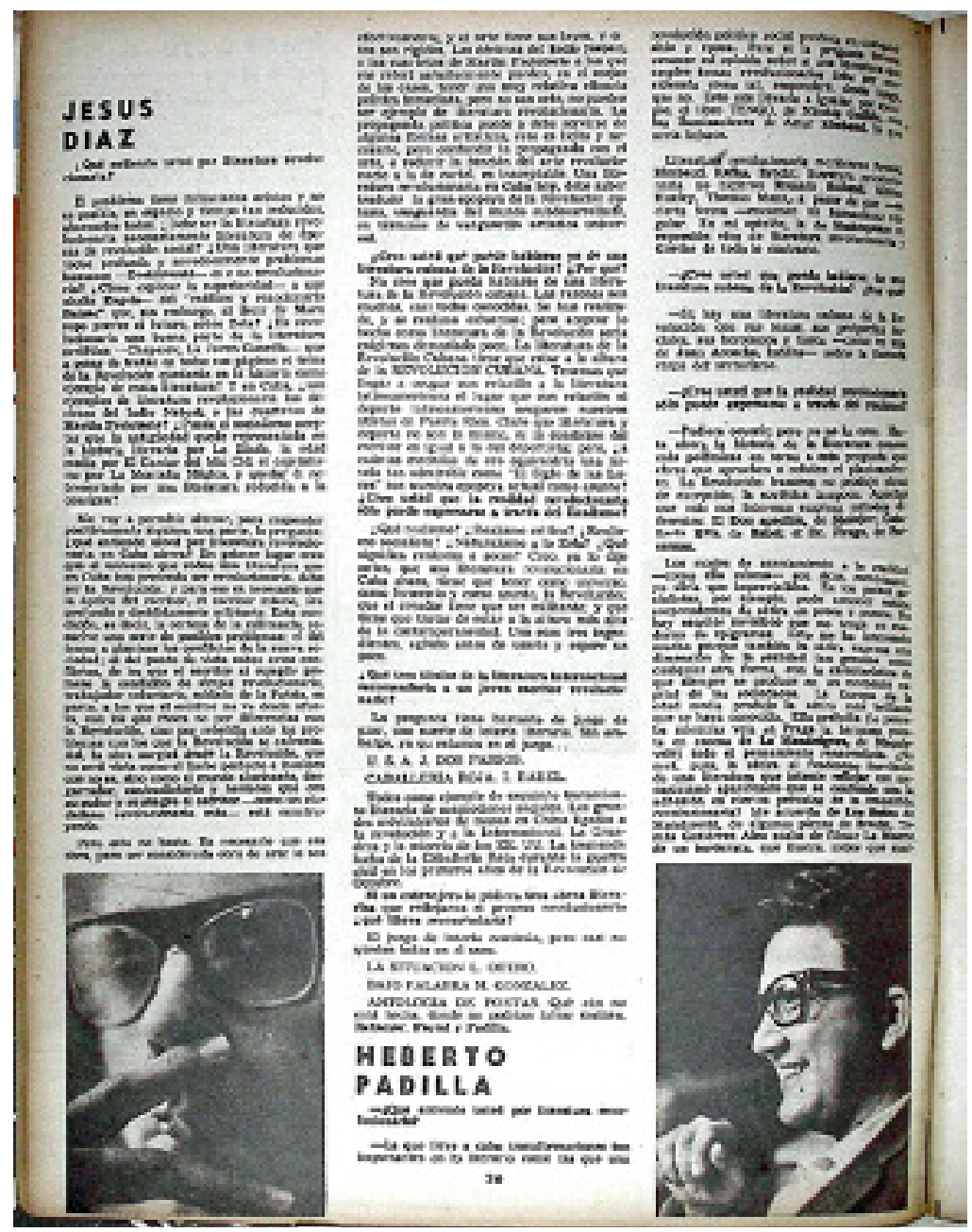

Fuente:https://www.annaillustration.com/archivodeconnie/wp-content/uploads/2007/06/Diaz-Nabori66.pdf Es en este contexto que las despectivas palabras con las que el joven director se refirió a la obra del reconocido poeta Jesús Orta Ruiz, conocido como "El Indio Naborí", generó una réplica de este en la edición de Bohemia del 5 de agosto, a la que Díaz contestó con un extenso ensayo el 16 de septiembre. En la encuesta, Díaz no solo se diferenció del resto de los entrevistados al ser el único en negar la existencia de una literatura revolucionaria en Cuba, sino que menospreció la obra de Naborí al considerarla un fallido producto del inmediatismo político carente de contenido estético: 
¿Son ejemplos de la literatura revolucionaria las décimas del Indio Naborí, o las cuartetas de Martín Proletario? ¿Puede el socialismo aceptar que la antigüedad quede representada en la historia literaria por la Ilíada, la edad media por El Cantar del Mio Cid, el capitalismo por La montaña mágica, y quedar él representado por una literatura reducida a la consigna? (...) Las décimas del Indio Naborí (...) pueden, en el mejor de los casos, tener una muy relativa eficacia política inmediata, pero no son arte, no pueden ser ejemplo de literatura revolucionaria ${ }^{10}$.

Dos semanas después se publicó la "Respuesta fraternal a Jesús Díaz”. Allí, el poeta le cuestionó la forma en que se remitió a su obra a la vez que le objetó procurar el destierro de la cultura popular cubana a manos de una experimentación estética influenciada por la cultura occidental: "Tú quieres, de un golpe, con la misma ira de los trovadores cortesanos contra los juglares, hacer desaparecer esa base, creyendo que puedes encontrarla en Londres, en Nueva York, en París, o en alguna de esas forzosas originalidades"11. Naborí asume que su obra se remite a un tiempo y espacio limitado por la urgencia política, pero lo justifica por un expreso pedido de la dirigencia revolucionaria en el marco de la lucha ideológica en curso:

Algunos jefes de la Revolución -y por cierto nada incultos- estimaron que mi versificación cotidiana podía ser útil como vía graciosa y sutil para llevar a nuestros campesinos y a nuestros obreros el mensaje de la Revolución (...). [C]omo me convencieron de que aquel trabajo era necesario, lo hice gustosamente, soltando el violín, que ya estaba bien afinado, y tomando nuevamente la humildísima bandurria $^{12}$.

Lo más relevante, sin embargo, fue la réplica de Díaz a través de un extenso escrito con visos programáticos en el que intentó trascender el debate personal para insertarse en el de las nociones estético-culturales necesarias en una Revolución. El título del texto, "Para una cultura militante", despersonalizó la discusión y la ubicó en el terreno de la reflexión sobre la construcción de una cultura ${ }^{13}$ socialista en Cuba.

Díaz dividió en tres apartados su escrito. Al primero lo tituló "Arte y capitalismo" y allí se detuvo en la conflictiva relación entre ambos debido, fundamentalmente, a la perenne resistencia del arte a ser valorado en términos cuantificables. A su vez, analizó el surgimiento de la denominada cultura de masas durante el siglo

10 Jesús Díaz, <<Literatura Revolucionaria>>, Bohemia Año 58 N² 29 (1966): 26.

11 Jesús Orta Ruiz, <<Respuesta fraternal a Jesús Díaz >>, Bohemia Año 58 Nº 31 (1966): 26.

12 Jesús Orta Ruiz, $<<$ Respuesta fraternal... $>>, 27$.

13 Si bien el concepto de "cultura" posee un más amplio sentido, aquí lo circunscribimos a la producción artística, literaria y teórica con el fin de remitirnos a los ejes del debate intelectual que recorren los posicionamientos de Jesús Díaz y del colectivo de El caimán barbudo en los textos a los que se aboca este trabajo. 
$\mathrm{XX}$, a la que fundamentó a partir de dos hechos conexos: la necesidad de la burguesía de instruir a vastos sectores de trabajadores para que desarrollen sus tareas laborales y la conquista de las ocho horas diarias de trabajo por parte de la clase obrera, lo que le otorgó mayor tiempo libre: "Estas dos cosas generan en la burguesía la posibilidad de desarrollar la cultura de masas. Convertirá así a los sectores populares en analfabetos políticos y ganará dinero. La cultura del entretenimiento pasatista"14. Lo relevante de esta mirada fue la conclusión de desvincular lo popular de lo masivo -y con ello replicar a las palabras de Naborí cuando lo acusó de pretender eliminar la cultura popular de Cuba-, ya que lo masivo muchas veces se encuentra teñido por el comercialismo y la impronta que la clase dominante ha dado a la práctica cultural. De allí derivó que la exitosa receptibilidad de una obra no es sinónimo de su identidad popular. Incluso encontró mayor peligro en el arte denominado realista o popular que en el de vanguardia ${ }^{15}$. El riesgo no estaría, por ejemplo, en el arte abstracto o en la dodecafonía musical -como establecía la crítica dogmática del momento, dentro de la cual podemos adscribir el pensamiento de Naborí en este debate-, sino en parte de la pintura costumbrista y en determinado realismo que nos espeja con modelos culturales, lenguajes y subjetividades que no se corresponden con los propios. De ahí dedujo que el gusto popular es también un espacio de lucha, como la conciencia política, por lo que en ocasiones lo masivo puede estar orientado por criterios y valores antagónicos a los que promueve la nueva sociedad que pretende construirse. Si los dogmáticos consideraban necesario apelar a formas estéticas estereotipadas y a ciertos criterios reconocidos para favorecer la comunicabilidad y utilizar el arte como mensaje o pedagogía, Díaz le endilgó a ello una peligrosa continuidad con el pensamiento hegemónico prerrevolucionario y, por ello, privilegió las experiencias de ruptura.

Díaz recuerda en su escrito que las formas realmente populares de la cultura cubana -como la décima en literatura o la música afro- llegaron desde el exterior y fueron absorbidas por el pueblo, quien las modificó en una hibridación creativa y enriquecedora. Esto le es útil para demoler los señalamientos de Naborí respecto de perseguir la base del nuevo arte nacional en producciones foráneas y, a la vez, para distinguir entre lo que considera una poesía popular y otra realizada por

14 Jesús Díaz <<Para una cultura militante >>, en Polémicas culturales de los años sesenta, ed. Graziella Pogolotti (La Habana: Letras Cubanas, 2006), 344.

15 Aunque en la tradición crítica las vanguardias suelen ocupar un espacio determinado del itinerario artístico de principios del siglo XX, en este trabajo retomamos la definición que surge de los dichos del propio Jesús Díaz, que considera "vanguardia artística" a toda aquella producción que prioriza el aspecto experimental y logra una originalidad procedimental en el terreno estrictamente estético que permite trascender lo producido hasta entonces. 
intelectuales que, con una presunción pedagógica, tratan de imitar lo reconocido por una comunidad para obtener mayor eficacia receptiva.

Será en el segundo apartado donde desarrolle esta concepción a partir de un epígrafe que fundamentará sus palabras: la frase del poeta español Antonio Machado según la cual "escribir para el pueblo es llamarse Cervantes en España, Shakespeare en Inglaterra, Tolstoi en Rusia"16. Díaz agrega: "Martí en Cuba". Por lo tanto: "Escribir para el pueblo conlleva ser vanguardia, expresar antes y en nuevos lenguajes el sentimiento popular. Otorga enormes posibilidades al desarrollo artístico, pues autores populares fueron Cervantes, Shakespeare, Tolstoi, Martí" ${ }^{17}$. La grandeza de estos autores estuvo en su originalidad estética y no en repetir moldes preestablecidos. Fueron quienes recuperaron creativamente una tradición y la actualizaron.

Aquí radica el giro fundamental del escrito de Díaz: fusionar los conceptos popular y vanguardia, generalmente diferenciados hasta el antagonismo por la tradición crítica, en una argumentación que rememora los planteos de Bertolt Brecht en la década del ' $30^{18}$ respecto del expresionismo y los debates entre herejes y dogmáticos de unos años atrás en la propia Cuba, pues los artistas nombrados por Machado, desde la perspectiva de Díaz, descubrieron nuevas realidades con los más afinados elementos artísticos y, con ello, se adelantaron a su época sin perder una óptica popular.

El pasaje que utilizó Díaz como epígrafe, a su vez, instauró una continuidad explícita con otros dos textos editados en Cuba durante esta etapa. El más cercano fue el Editorial con el que se inició el primer número de El caimán barbudo, publicado apenas cuatro meses antes de la aparición del texto de Díaz en Bohemia. Allí los caimanes -por supuesto, con Díaz a la cabeza- utilizaron el mismo fragmento de Machado en su presentación oficial. Con ello, el autor de Los años duros (1966) integró sus dichos en Bohemia en el marco del colectivo cultural que lo incluía, el de la revista que comenzó a dirigir y que pretendía representar a la juventud revolucionaria cubana. Pero, a su vez, como nota Martínez Pérez, el pasaje de Machado también había sido empleado por el presidente de Cuba, Osvaldo Dorticós Torrado, durante la inauguración del congreso fundacional de la UNEAC en agosto de 1961, con lo que se evidencia la intención de identificarse no solamente como parte de una nueva camada que surge en Cuba, sino a su vez con el histórico liderazgo político de la Revolución. Desde esos lugares el autor le devuelve irónicamente a Naborí su metáfora del violín:

16 Jesús Díaz $<<$ Para una cultura... $>>, 347$.

17 Jesús Díaz $<<$ Para una cultura... $>>, 347$.

18 Ver Bertolt Brecht, El compromiso en literatura y arte (Barcelona: Península, 1984). 
No se trata de soltar el violín, se trata de tener confianza en las capacidades populares para escucharlo, de tener conciencia de la situación a que estuvo sometido el pueblo. Se trata de creer realmente en la fuerza del pueblo, de hablar francamente al pueblo. De juntos, creador y pueblo, aprender éste a tocar mejor el mejor instrumento, aquél a escuchar mejor el mejor instrumento. De juntos producir la mejor música, a la que el pueblo tiene derecho. Escribir para el pueblo significa en primer lugar respetarlo ${ }^{19}$.

Pero Díaz también se permitió problematizar lo que se considera popular desde otro aspecto al abordarlo como un concepto dinámico. Los pueblos se transforman permanentemente, a veces incluso se revolucionan, y lo que en un momento fue popular -la décima, por ejemplo- puede dejar de serlo en un futuro o en el presente. Del mismo modo, algo que no lo era puede convertirse en popular tiempo después gracias a relecturas, nuevos contextos, cambios en la subjetividad y el gusto estético, entre otras variables. Por ello llamó a evitar anquilosarse en rígidas formas antiguas, sobre todo en Cuba, pues si este pueblo ha cambiado notoriamente en la última década gracias a la Revolución, de ningún modo lo popular podría mantenerse inmutable.

Es desde aquí que pretendió exponer lo que consideró las tareas del intelectual cubano en su contemporaneidad: producir un arte de vanguardia popular y revolucionario acorde a un contexto nacional caracterizado por el subdesarrollo socio-económico y la transición al socialismo, un arte que sea expresión de su comunidad en su tiempo y no una marca de aislamiento o distinción: "El centro de la problemática intelectual de Cuba -si del trabajo de los creadores se trata- (...) es traducir la experiencia de la Revolución Cubana, vanguardia del mundo subdesarrollado, en términos de vanguardia artística ${ }^{20}$. Asimismo, esta vanguardia popular en revolución no debe pensarse como reiteración de fórmulas experimentales previamente adquiridas, pues los procedimientos que fueron vanguardistas en un momento histórico pasado ya forman parte del acervo tradicional del arte. La vanguardia, como su denominación lo sugiere, debe estar delante del autor y no a su espalda, por lo que debe fortalecerse la creatividad, la inventiva y la experimentación a partir de la absorción del acervo cultural universal y de la tradición local en busca de nuevas síntesis.

19 Jesús Díaz, $<<$ Para una cultura... $>>, 347$.

20 Jesús Díaz, $<<$ Para una cultura... $>>, 350$. 
Figura 3. Portada de El caimán barbudo del N 17, de octubre-noviembre de 1967

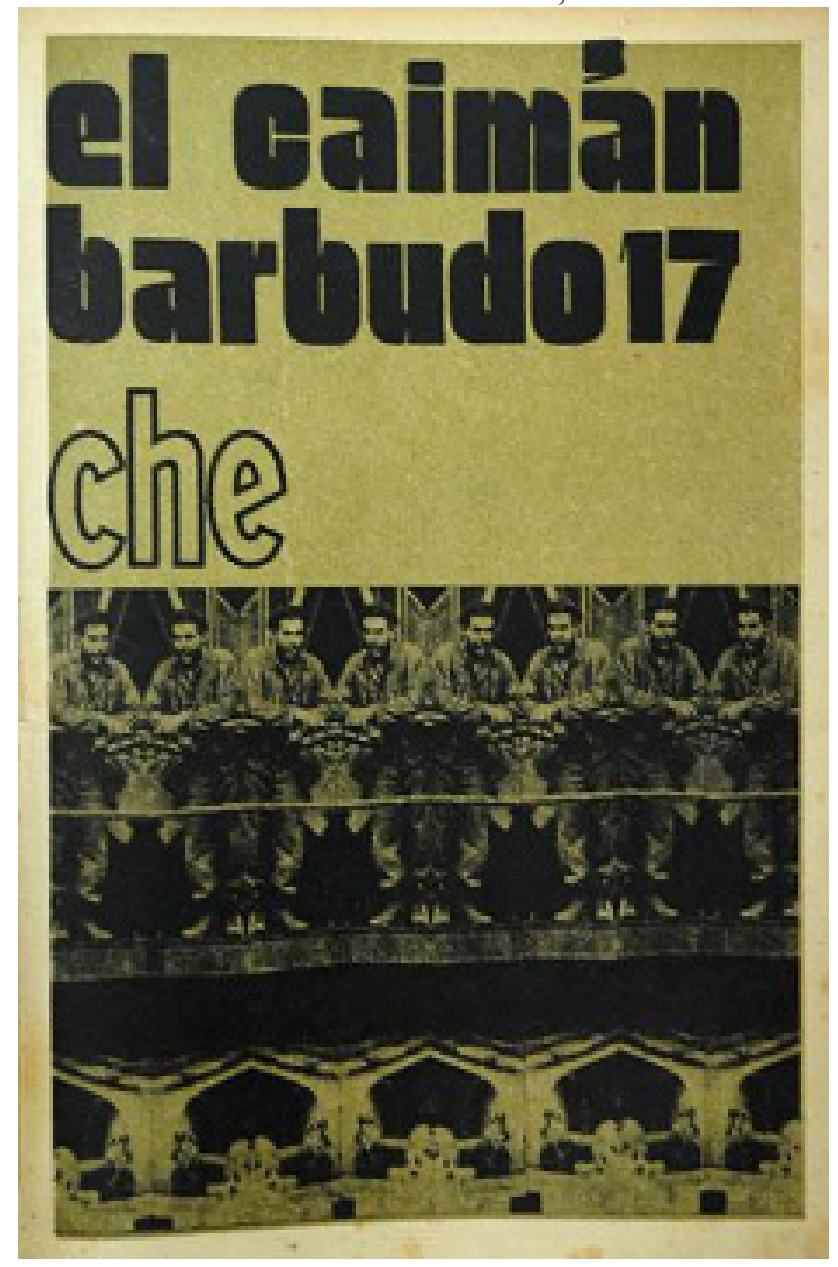

Fuente:http://www.caimanbarbudo.cu/entrevistas/2011/06/\%C2\%BFcuando-se-publico-el-primer-caiman-barbudo/

De esta manera, el director de El caimán barbudo se orientó hacia una articulación entre vanguardia política ${ }^{21}$ y vanguardia estética. Si dentro de la Revolución, todo, como propuso Fidel en 1961, el creador revolucionario no tendría nada que perder: "con la Revolución la alegría y la tristeza y sobre todo el futuro. El futuro de un mundo donde el hombre no es más simple, sino más complejo; no más ignorante, sino más culto; no más irracional, sino más crítico y más consciente"22. En este proceso se requiere una amplia política cultural emancipadora -liberada

21 El concepto de "vanguardia" utilizado en términos políticos replica aquí la caracterización de los miembros de El caimán, según la cual la vanguardia es la dirección política del proceso revolucionario.

22 Jesús Díaz, $<<$ Para una cultura... >>, 356. 
de las fórmulas reguladoras del arte como la realista socialista- que no refleje meramente necesidades inmediatas ajenas a lo inherentemente artístico. El arte debe huir de la propaganda y encontrar su propio espacio y funcionalidad dentro de la Revolución como tal, es decir, como arte: "El arte de vanguardia no entendido por todo el mundo tiene derecho a existir como toda investigación, como todo aporte. La aspiración debe ser convertirlo, si es bueno, en un arte de masas lo más pronto posible"23. Nuevamente, nunca "soltar el violín", sino generar las condiciones para su mayor y más profunda recepción.

Para ello, el rol de los funcionarios debe distar hasta el antagonismo del que orientó otras experiencias socialistas como la de la URSS, en donde la dirigencia política coartó el desarrollo cultural al imponer una estética oficial regida por parámetros inmediatistas, pedagógicos y comunicativos:

Un arte popular de vanguardia es posible; elcine, máximo aporte cultural de la Revolución Rusa en los primeros años, lo ha probado. Eisenstein, un comunista, es tan inventor del cine como Lumière. (...) Mayakovski, el gran poeta de la Revolución, fue, es, querido, escuchado, por las masas. Fue también un revolucionario de la poesía. Ejemplos sobran y sobrarían aún más si tantos burócratas de la cultura no se hubieran metido entre el pueblo y los creadores dictando normas a diestra y siniestra; identificando -como dice el Che- lo que ellos entienden con lo que entiende el pueblo ${ }^{24}$.

Estos dichos coincidieron con textos colectivos publicados en El caimán, como por ejemplo el ya nombrado Editorial del primer número, en el que se sitúa al magazine como obra de jóvenes revolucionarios conscientes tanto de su militancia como de su alejamiento de toda clase de dogmatismo que ponga freno al desarrollo de la cultura. Este texto expresó la intención de evitar el pensamiento y la práctica prefijados, y alentó la experimentación y la investigación sin horadar el espíritu y la actitud militante dentro del proceso revolucionario socialista cubano. A su vez, al citar a Machado se lo imbricó con textos de Lenin, Martí y Castro. La cultura universal, la política revolucionaria, la tradición política y cultural local y el presente de la Revolución Cubana se retroalimentaban desde esta visión:

Creemos, con Machado, que escribir para el pueblo es llamarse Cervantes en España, Shakespeare en Inglaterra y Tolstoy en Rusia; creemos, con Lenin, que la cultura nueva sólo puede ser desarrollada conociendo con precisión la cultura que ha creado la humanidad en toda su historia y transformándola; creemos, con Martí, que no hay igualdad de derechos sin igualdad de cultura; creemos, con Fidel, que debe ser preocupación fundamental de la Revolución el desarrollo espiritual del hombre nuevo ${ }^{25}$.

23 Jesús Díaz, $<<$ Para una cultura... >>, 358-59.

24 Jesús Díaz, $<<$ Para una cultura... $>>, 358$.

$25<<$ Editorial $>>$, El caimán barbudo $\mathrm{N}^{\circ}$ 1, (1966): 1. 
En este pasaje se realizó algo más que utilizar ciertos nombres de autoridad en los que apoyarse. Toda una política cultural se desplegó a partir de aquí. En primer lugar, tal como poco después de la publicación de este Editorial expresará Díaz en el debate con Naborí, se estableció que el arte no debe rebajar su calidad y experimentación para convertirse en popular. No hay escritor más representativo en España que Cervantes, ni en Inglaterra que Shakespeare, ni en Rusia que Tolstoi; los cuales trascendieron su período contemporáneo de recepción. Si ellos se convirtieron en referentes de sus respectivas culturas nacionales y en artistas universales a la vez, fue porque produjeron una obra que representa tanto lo peculiar de su pueblo como la continuidad de la cultura más allá de cualquier frontera.

La mención de Lenin en relación con la necesidad de conocer, estudiar y retomar creativamente la cultura universal para desarrollar un cambio civilizatorio socialista, halló en uno de los clásicos del marxismo agua para llevar al molino propio. Así como no hay forma de construir una nueva cultura desde la nada, tampoco solamente a partir de la establecida por la tradición socialista. Hay que asimilar la producción cultural de toda la historia y crear desde allí algo nuevo, es decir, transformarla. El verdadero leninismo debe asumir este principio, contrario al dogmatismo de la impronta soviética de entonces. La frase de Martí, por su parte, permite afirmar que la lucha de liberación y la de la construcción del socialismo distan de ser meros intentos de emancipación política y soberanía económica. Sin igualdad en el acceso a la cultura -por lo tanto, podríamos agregar, sin funcionarios que seleccionen lo que las masas deben leer, ver o escuchar-, no hay igualdad civil. Por lo tanto, no hay desarrollo colectivista y socializador. Finalmente, y ligado a ello, aparece el pasaje de Fidel con notorias reminiscencias a Ernesto Guevara a poco más de un año de la aparición de El socialismo y el hombre en Cuba (1965), texto en el que se promueve la construcción del "hombre nuevo" como necesidad primordial de la Revolución.

Por eso el vínculo entre este texto colectivo y el posicionamiento de Díaz en "Para una cultura militante" (1966) resulta notorio. El director de El caimán se ubicó como interlocutor de una concepción estética y política sostenida por los nuevos intelectuales y artistas de Cuba en discordancia con lineamientos de otras tendencias político-culturales presentes dentro de la Revolución, como por ejemplo la denominada dogmática expresada en este debate por Naborí. Además, se observa en ambos textos la intención de posicionarse retomando al máximo liderazgo del proceso, como Dorticós, Fidel y el Che. 


\section{La generación y la revolución}

Otra de las polémicas del año '66 se dio entre el propio Díaz y la antigua co-responsable de Ediciones El Puente -Ana María Simó- entre los meses de abril y agosto. En el número 50 del año V de La Gaceta de Cuba, perteneciente a abril y mayo, se divulgaron una serie de textos de intelectuales cubanos respecto de la problemática de las generaciones intelectuales en el país. Bajo el título "Encuesta sobre las generaciones" Carpentier, Félix Pita Rodríguez, José Lezama Lima, Portuondo, Fernández Retamar, Otero, César López, Miguel Barnet, Nicolás Dorr y Díaz respondieron tres preguntas: ¿Qué entiende usted por confrontación generacional? ¿Cree usted que después de 1959 en Cuba esa confrontación tiene razón de ser? ¿Cómo definiría usted su generación? Como le sucederá con la encuesta de Bohemia, los dichos de Díaz suscitaron una enconada réplica, esta vez debido a sus críticas a la producción de la Editorial El Puente que funcionó en Cuba entre 1961 y 1964. Simó publicó su respuesta en el número siguiente de $L a$ Gaceta, correspondiente a los meses de junio y julio, y finalmente Díaz contestó en el de los meses de agosto y septiembre. Es decir, esta pelea se desplegó prácticamente en simultaneidad a la disputa de Díaz con Orta Ruiz.

Díaz explicitó que una generación nunca es homogénea y que luego del triunfo revolucionario existen campos de confrontación más fuertes que las líneas generacionales, como por ejemplo los ideológicos. Cuestionó la labor de los intelectuales dentro de la Revolución debido a lo que denominó su incapacidad crítica y se detuvo en los jóvenes que confluyeron en la Editorial El Puente por considerarlos la mayor expresión contemporánea del pensamiento liberal en la isla. Así, definió a su generación -a partir de un uso tradicional y acrítico del término ${ }^{26}$ - como fragmentada en dos perspectivas antagónicas: la de El Puente, y, sin explicitarla, la de El caimán.

Simó refutó las posturas de Díaz al afirmar la inexistencia de un grupo homogéneo en El Puente, a la vez que reivindicó el trabajo realizado durante los cuatro años de existencia de la editorial. Para ella lo único que unió a los integrantes de aquel proyecto fue: "mantener abierta una oportunidad de expresión para los jóvenes escritores, sin discriminaciones de escuela literaria. (...) El papel de las Ediciones

26 En su libro La nueva generación intelectual. Incitaciones y ensayos (Buenos Aires: Herramienta, 2008), el historiador argentino Omar Acha elaboró una concepción de "generación intelectual” que resulta más provechosa al afirmar que una generación no solo ni sustancialmente puede verificarse en torno a lo etario, así como tampoco por la yuxtaposición de individuos aislados; sino prioritariamente por pertenecer a una determinada época histórica y formar parte de un trazado colectivo -lo cual no necesariamente implica pertenencia a un específico grupo- a partir de una sensibilidad innovadora que opera sobre una realidad material concreta. En parte cercano a una tradición que se remonta al pensamiento de Mannheim y adherido a la concepción gramsciana, para Acha la edad de los miembros de una generación -excluyente en la propuesta de Díaz y en la Encuesta en general-, se establece como un factor contingente. 
fue, por tanto, más el de una empresa práctica que estética e ideológica"27. Luego ubicó como autoridad absoluta de la editorial -y como responsable de su rumbo en los últimos tiempos- al ya por entonces emigrado poeta José Mario Rodríguez, con lo que le endilgó a Díaz una confusión entre la actitud individual de este y la significación histórica del proyecto.

Si bien asumió cierta ingenuidad de los integrantes de El Puente y juzgó el repliegue intelectual sobre sí mismos que se expresó a partir del año 1962 como un exceso de protección hacia la obra propia, lo justificó a raíz del contexto en el que se generó, dominado por el Proceso al sectarismo. Entiende que ello derivó en una sistemática desconfianza hacia la política y en un temor a convertir las obras en panfletos. Sus búsquedas, en definitiva, las resumió en la promoción de la participación de todos los jóvenes creadores y en aceitar los nexos entre los miembros de su propia generación.

Figura 4. Respuesta final de Díaz a Simó publicada en el N 52 de La Gaceta de Cuba.

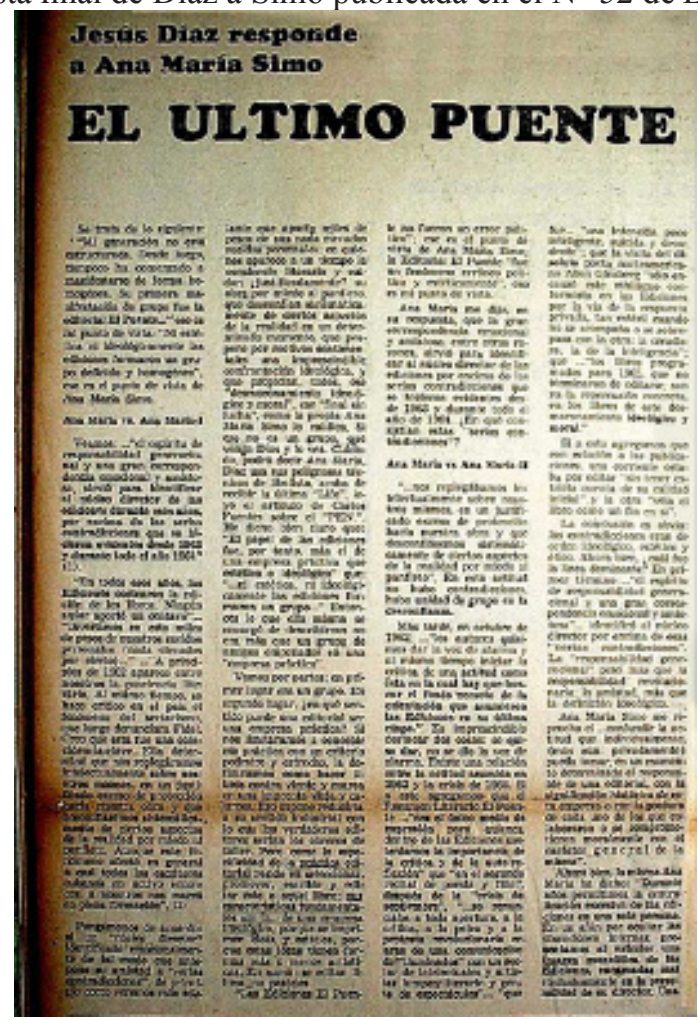

Fuente: https://www.annaillustration.com/archivodeconnie/wp-content/uploads/2007/04/J.Diaz_contesta.pdf

27 Ana María Simó, <<Respuesta a Jesús Díaz>>, en Polémicas culturales..., 370. 
Un número después llegó la respuesta de Díaz -"El último puente"- en la que una a una fue desmoronando las fundamentaciones de Simó. Primero, le rebatió la consideración de la inexistencia del carácter grupal de la editorial, y lo hizo retomando textuales palabras de la autora para defender su postura:

Pongámonos de acuerdo: si un núcleo director identificado emocionalmente de tal modo que antepone su amistad a serias contradicciones, de principio como veremos más adelante; que aporta miles de pesos de sus nada elevados sueldos personales; en quienes aparece a un tiempo la conciencia literaria y cuidan ijustificadamente? su obra por miedo al panfleto, que desconfían sistemáticamente de ciertos aspectos de la realidad en un determinado momento, que pospone por motivos sentimentales una imprescindible confrontación ideológica, y que propician, todos, ese desmoronamiento ideológico y moral, ese final sin lucha, como la propia Ana María Simó lo califica. Si eso no es un grupo, que venga Dios y lo vea ${ }^{28}$.

Luego cuestionó la noción de que una editorial sea una empresa meramente práctica, como si se tratase de un hecho industrial más. Promover, escribir y editar libros es un proyecto intrínsecamente ideológico. Por otra parte, no observó contradicciones entre las posiciones del grupo en sus comienzos y lo que motivó su final. Su definitivo cierre en 1964 fue el resultado lógico de aquel repliegue hacia sí mismos que comenzó en 1962. El grupo tuvo unidad en la desconfianza hacia el proceso político, esa fue su línea dominante, sostenida por un espíritu generacional en términos meramente etarios y en una correspondencia amistosa por encima de diferencias políticas. Así, Díaz no solamente se diferenció del dogmatismo por establecer una subordinación del hecho estético ante las urgencias políticas, como se observa en su disputa con Orta Ruiz, sino que a la vez se delimitó de aquellos que pretendían producir un arte ajeno al -o por fuera del- contexto revolucionario, perspectiva que le endilga aquí a Simó.

Otro de los textos colectivos publicados en el primer número de El caimán barbudo (1966), el Manifiesto "Nos pronunciamos" firmado por catorce poetas y convertido en programático en esta etapa hasta el punto de articular una propuesta estética revolucionaria dentro de Cuba, se había posicionado en las antípodas de cualquier tipo de práctica estética que eludiera su contexto social y político. Allí los poetas fijaron una distinción fundamental entre su generación y las previas que se integraron al proceso revolucionario luego del 1 de enero de 1959 precisamente en este aspecto. Si aquellas habían sido más o menos ajenas a la insurrección y recién se sumaron al proceso revolucionario luego de su triunfo, para los jóvenes integrantes de El caimán: "La revolución no llegó a nosotros como a gente formada a su margen: trece años de nuestra vida -sin duda los

28 Jesús Díaz, <<El último puente>>, La Gaceta de Cuba Año V Nº 52 (1966): 4. 
más importantes- han sido los años de la Revolución combatiente y vencedora" ${ }^{29}$. El principal motivo de unión, por lo tanto -en sintonía con lo que propuso el Editorial inaugural- no lo conforman lineamientos meramente estéticos comunes -como a las publicaciones prerrevolucionarias- ni un evento político ajeno -como a los que se sumaron a la Revolución pos 1 de enero de 1959-, sino la integración al proceso político en curso. Ellos no se suman a la Revolución, son sus hijos. Esto tiene como conclusión el que la escritura que despliegan es realizada desde el propio proceso revolucionario, no para el proceso, sino desde su interior: "No podemos ser, pues, gente presta o negada a adecuar su voz a la Revolución. Con ella nos hemos formado -nos estamos formando-, sin ella no podríamos explicarnos" ${ }^{\prime 3}$. Los poetas explicitan que son la primera generación revolucionaria de Cuba. Por ende, son su expresión más auténtica: "No pretendemos hacer poesía a la Revolución. Queremos hacer poesía de, desde, por la Revolución" "31. Se observa, así, el abismo que separaba esta concepción de la experiencia de El Puente descripta por Simó.

Acto seguido, tal como lo realizó el Editorial, este Manifiesto posicionó la práctica cultural del grupo de poetas en su contexto de país del Tercer Mundo y apuntó que el desarrollo nacional y la autenticidad cultural se interrelacionan de tal modo que la construcción del comunismo es condición para el logro de ambos objetivos, lo que Martínez Pérez llama un convencimiento de que el futuro de Cuba depende de su desarrollo cultural, pues allí radica la posibilidad de una concreta liberación humana.

29 Orlando Alomá et al., <<Nos Pronunciamos $>>$, El caiman barbudo $\mathrm{N}^{\circ} 1$ (1966): 11.

30 Orlando Alomá et al., $<<$ Nos Pronunciamos $>>$, 11.

31 Orlando Alomá et al., $<<$ Nos Pronunciamos $>>, 11$. 
Figura 5. Manifiesto "Nos pronunciamos", publicado en el $\mathrm{N}^{\circ} 1$ de El caimán barbudo

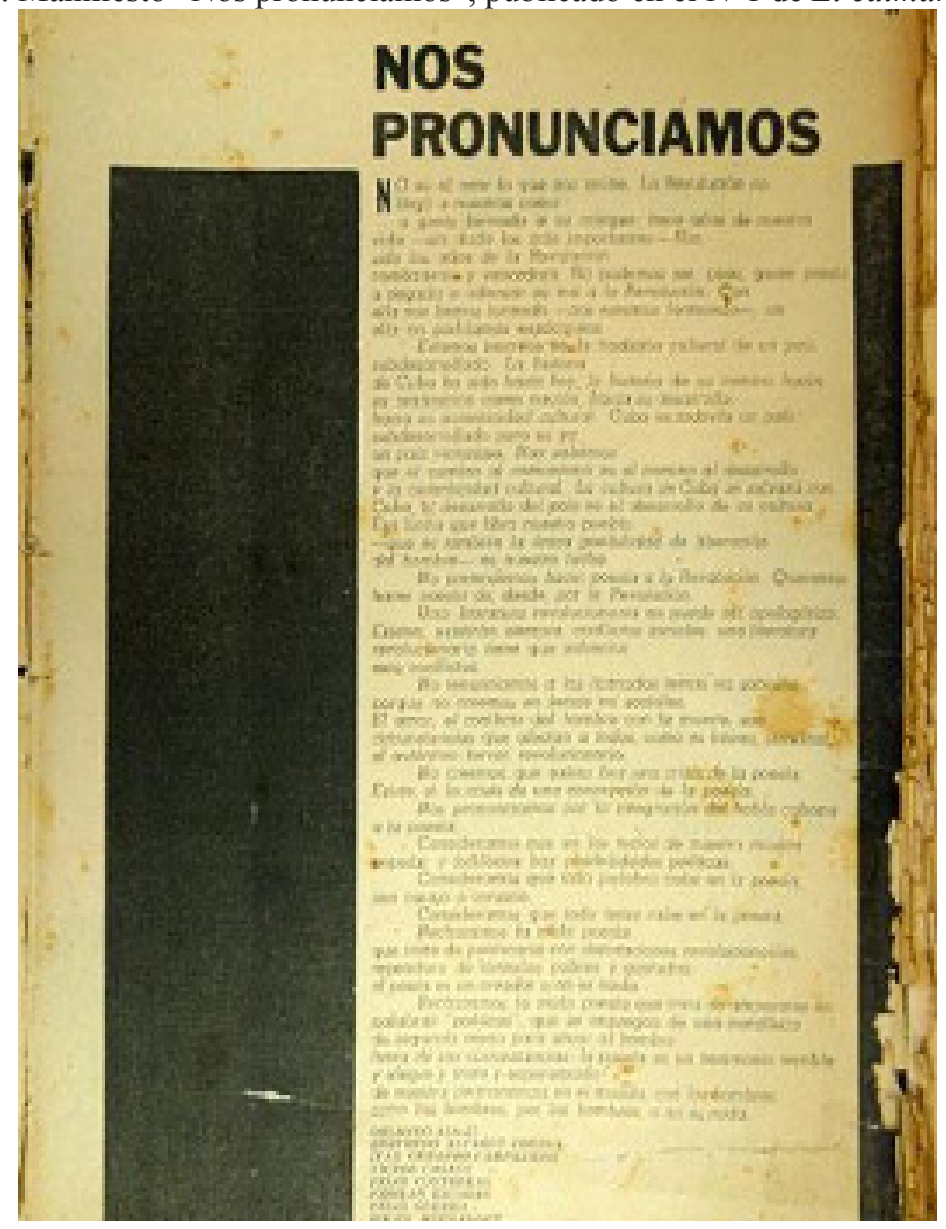

Fuente:http://www.caimanbarbudo.cu/entrevistas/2011/06/\%C2\%BFcuando-se-publico-el-primer-caiman-barbudo/

Por último, el texto se abocó a particularidades de la poesía. Un arte crítico que hable de la realidad de su tiempo -por ende, de la Revolución-, que asuma la tradición previa y la transforme a través de la experimentación es la propuesta de estos poetas, que así como amparados en la máxima de que el poeta es un creador o no es nada, rechazaron el arte que se justifica en denotaciones revolucionarias; también impugnaron a quienes omiten su contexto: "la poesía es un testimonio terrible y alegre y triste y esperanzado de nuestra permanencia en el mundo, con los hombres, por los hombres, o no es nada"32. Se distinguieron así de los extremos que se hallan en los textos de Naborí y de Simó: ni subordinación política

32 Orlando Alomá et al., $<<$ Nos Pronunciamos $>>, 11$. 
ni evasión. Nuevamente, se evidencia que las polémicas que protagonizó Díaz no se correspondieron con un señalamiento individual, sino que se integraron a lineamientos de una corriente de pensamiento.

\section{El artista como funcionario}

Las polémicas continuaron a lo largo de todo el breve itinerario de la primera época de El caimán barbudo. Menos de un año después de las querellas con Naborí y Simó, en el número 15 del magazine perteneciente al mes de junio de 1967 se dio un debate entre el Consejo de Redacción y Heberto Padilla que se convirtió en el detonante para el final del ciclo de Díaz al frente del proyecto. El conflicto se generó a raíz del pedido que los miembros de El caimán realizaron a Padilla para que colaborara con un comentario sobre la última obra de Lisandro Otero, Pasión de Urbino (1966). Padilla envió un escrito en el que sugirió que los integrantes de El caimán privilegiaron el análisis de esa novela por razones políticas y no estéticas, ya que Otero era el vicepresidente del CNC. Eso mismo los habría llevado a desconocer la aparición de Tres Tristes Tigres (1967), de Guillermo Cabrera Infante, a la que consideró: "sin duda, una de las novelas más brillantes, más ingeniosas y profundamente cubanas que hayan sido escritas alguna vez" ${ }^{33}$-. El rol político de Otero dentro de la isla y la decisión de Cabrera Infante de emigrar hacia Europa serían, según esta lectura, los argumentos para que la pregunta fuese sobre el texto de Otero y no sobre el del antiguo director de Lunes de Revolución, pues entre una y otra novela se presentaría un indiscutible ejemplo de la diferencia entre el talento literario y la ramplonería. Por lo tanto, acusa al Consejo de Redacción de El caimán de someter una posición estética a una presión política.

33 Heberto Padilla, <<A propósito de Pasión de Urbino >>, El caimán barbudo N 15 (1967): 12. 
Leonardo Candiano

Los primeros dientes de El caimán barbudo (1966-1967)

Figura 6. Portada de la novela Pasión de Urbino, de Lisandro Otero.

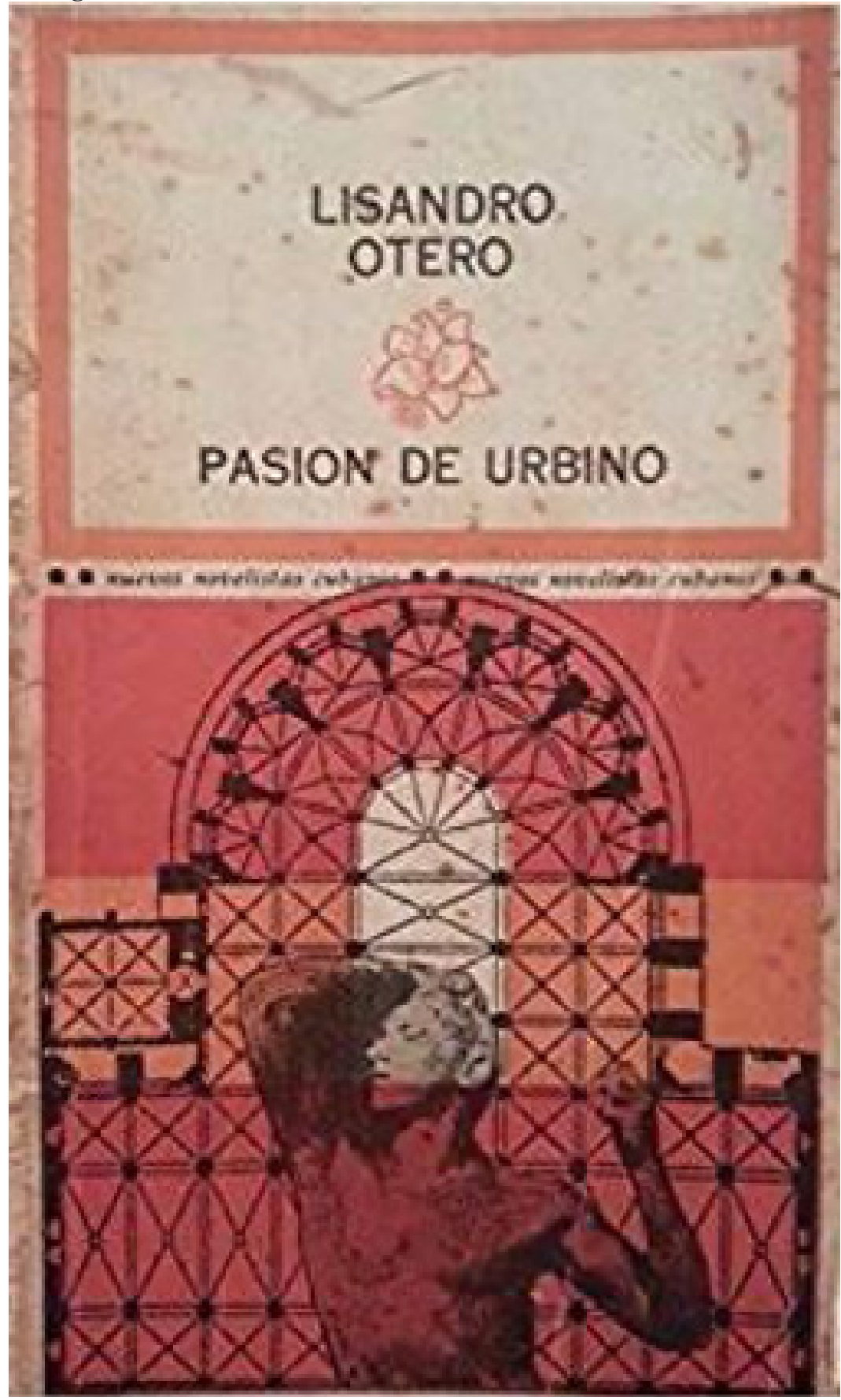

Fuente: http://www.lajiribilla.co.cu/2007/n301_02/301_63.html 
Aunque no haya respondido la demanda del magazine, el artículo fue igualmente publicado junto con un desacuerdo firmado por el Consejo de Redacción. Los caimanes replicaron que la heterogeneidad y la autonomía en el trabajo cotidiano resultan valores palpables dentro del ambiente cultural cubano y en la revista en particular, por lo que las definiciones de Padilla carecen de sustento, desconocen la realidad del país y solo pueden entenderse por la ausencia del poeta de la vida cultural cubana en los últimos años debido a su estadía en la URSS.

En lo que respecta al Consejo de Redacción el interrogante respecto de Cabrera Infante no debería ubicarse en un hipotético silenciamiento de su obra en las páginas de El caimán, sino en los fundamentos que lo llevaron a irse de Cuba -"ipor qué Cabrera Infante se encuentra hoy en un sótano de Londres y no en su casa del Vedado, escribiendo y trabajando para su país, para sus propios lectores, como lo hacen los escritores de la Cuba revolucionaria?" ${ }^{34}$, se preguntaron desde el mensuario. La respuesta que encontraron es que prevaleció en el autor un interés individual de desarrollo autoral en el exterior por sobre el compromiso colectivo en la dura construcción cotidiana de una sociedad nueva. Sin embargo, el énfasis del texto que representó la posición editorial se ubicó en el cuestionamiento a la dicotomía establecida por Padilla entre: "el gris burócrata de la cultura, que a duras penas podrá escribir divertimentos, o el del escritor revolucionario que se plantea diariamente su humilde, grave y difícil tarea en su sociedad y en su tiempo"35. Por el contrario, desde el magazine se consideró que ser funcionario cultural del Estado revolucionario era una oportunidad histórica para desplegar todo el talento creador que puede tener una persona en una obra de carácter social, ya que abarca tanto la escritura como el desarrollo de un movimiento cultural de masas:

En una sociedad desarrollada, miles de cuadros intermedios relevarían al creador de las responsabilidades del divulgador y el maestro. Pero en una sociedad subdesarrollada, que lucha confiada y ferozmente por salir del subdesarrollo, el creador tiene el privilegio y el deber del heroísmo: ha de crear simultáneamente su obra y el público capaz de disfrutarla y apreciarla. No se trata ya de escribir una buena novela, sino de crear un auténtico movimiento literario -digno de una de las revoluciones más profundas y dinámicas del mundo moderno- y un público activo para ese movimiento. Esto es algo que no puede hacerse desde un sótano de Londres, ni de París, ni de ninguna parte. Ha de hacerse aquí, por intelectuales y artistas revolucionarios. Por eso resulta sorprendente que Padilla hable de los dos caminos que se le ofrecen hoy al escritor cubano (...). Aquí, en este país, ser hoy "funcionario" de la Revolución en el campo cultural -es decir, poner su inteligencia, sus energías, su capacidad y su entusiasmo al servicio del desarrollo cultural de las masas- es uno de los más hermosos desafíos que puede hallar un escritor en el mundo contemporáneo. Es, en realidad, la única forma

34 Consejo de Redacción, El caimán barbudo $\mathrm{N}^{\circ} 15$ (1967): 13.

35 Heberto Padilla, <<A propósito de Pasión de Urbino >>, El caimán barbudo N 15 (1967): 12. 
revolucionaria de asumir la humilde, importante, difícil misión del escritor en su sociedad y en su tiempo ${ }^{36}$.

En este sentido, la defensa del rol de funcionario para el intelectual cubano -que por entonces detentaba Lisandro Otero- y la necesidad de habitar en la isla e inmiscuirse en la gestión estatal de los productos culturales -en lugar de expatriarse como Cabrera Infante- se trazó a partir de la capacidad que puede detentar un funcionario para articular el esfuerzo individual de los artistas y promover las potencialidades creativas en un número cada vez más amplio de personas.

Otero reveló en su libro Llover sobre mojado. Memorias de un intelectual cubano (1957-1997) (2001) que la polémica quedó suspendida por una decisión política. La inminencia del Congreso Cultural de La Habana de enero de 1968 -y su seminario preparatorio de finales del '67-, junto con el asesinato del Che Guevara en Bolivia, llevaron a un impasse en el debate; profundizado por la desarticulación del Consejo de Redacción original de El caimán en el mes de octubre de 1967 y su reemplazo por otro completamente nuevo con posiciones antagónicas que derivó, entre otras cosas, en una momentánea pérdida de la periodicidad del mensuario. Todo ello generó que 9 meses después, en el número 19 del mes de marzo de 1968, se publicara la respuesta de Padilla a la "Redacción saliente" de El caimán. Allí el poeta enfatizó la diferencia existente, según su perspectiva, entre el funcionario cultural y el creador artístico, pues si el primero debe necesariamente adscribirse a una razón de Estado, el segundo no puede ser otra cosa que la cabeza crítica del cuerpo social. El primero se asimilaría a un rutinario burócrata, el segundo a un librepensador.

Finalmente, en el número 21 perteneciente al mes de junio del ' 68 la "Redacción saliente" respondió con un nuevo artículo firmado por Díaz, Rodríguez Rivera, Casaus y Nogueras. El texto discutió la función social y la actitud del intelectual revolucionario mediante una aguda crítica a lo que consideraron el falso dilema expresado por Padilla con su tajante separación entre la acción cultural y la política. Para los firmantes la opción presentada por Padilla no solo es equivocada sino reaccionaria, ya que no debe existir necesariamente una oposición entre funcionarios y artistas. Los -ahora ex-caimanes reiteraron la ligazón orgánica entre ambos roles dentro de la realidad cubana.

La fusión entre las vanguardias artística y política en Cuba, y la asunción para ello de una nueva actitud crítica y militante por parte del intelectual, fueron los pilares que sostuvieron las nociones vertidas por la antigua redacción de El caimán aquí, en sintonía con las palabras de Díaz en "Para una cultura militante" y por el grupo de poetas en "Nos pronunciamos". No existen senderos que se

36 Consejo de Redacción, El caimán barbudo N 15 (1967): 13. 
bifurcan entre el gestor estatal de la producción cultural y el creador artístico, sino entrecruzamientos -a veces, incluso, encrucijadas- que deben transitarse sin recetas ni prejuicios.

\section{La mordida de El caimán}

En síntesis, el surgimiento de El caimán barbudo como una de las expresiones culturales de la nueva camada intelectual cubana continuó y ahondó uno de los rasgos inherentes del proceso revolucionario en sus inicios: su heterogeneidad interna y su afán polemista en el marco de la unidad política.

Esta situación tomó más ímpetu a partir del lugar preponderante que se le otorgó como parte de la dirección ideológica en la isla y por su vínculo orgánico con el liderazgo revolucionario, algo que puede afirmarse no solamente por la experiencia del magazine como órgano oficial de la UJC, sino también por la aparición en 1967 de Pensamiento Crítico y por la fundación del Instituto Cubano del Libro, ambos proyectos dirigidos por integrantes del Departamento de Filosofía de la Universidad de La Habana, así como por el rol de miembros de este Departamento en la Conferencia Tricontinental, en la OLAS y en la organización del Congreso Cultural de La Habana ${ }^{37}$.

Los debates de Jesús Díaz con Orta Ruiz y con Simó, al igual que el del Consejo de Redacción de El caimán con Padilla, fomentaron el despliegue de una intelectualidad popular y vanguardista orientada a resolver los problemas políticos y culturales que presenta el mundo subdesarrollado en plena revolución. Esa ligazón entre arte y política los llevó a cuestionar severamente prácticas que distanciaban ambas facetas, como por ejemplo la establecida por la Editorial El Puente, así como también las justificaciones de Padilla en su obstinada diferenciación entre funcionarios y artistas. Sin embargo, ello no derivó en una actitud de subordinación del hecho cultural ante el inmediatismo político -que se expresa en la perspectiva de Naborí- y, lejos del mecanicismo dogmático, en esta época los caimanes defendieron la experimentación como inherente a la producción estética, la cual debe regirse por reglas propias y bucear en su tradición peculiar. Solo así el vínculo entre arte y política podrá volverse eficaz en el marco de la construcción de una nueva cultura. Resulta notorio, a su vez, que esa autonomía necesaria para la práctica artística fue pensada desde el interior de la Revolución y nunca desde el requerimiento de externidad que insinuó indispensable el posicionamiento de Padilla.

37 Para profundizar en este aspecto ver Liliana Martínez Pérez, Los hijos de Saturno ...; Néstor Kohan, "Pensamiento Crítico..." 
Dentro del proceso revolucionario, los referentes de El caimán -principalmente Díaz- reflexionaron sobre la posibilidad que se le abría a los artistas para asumir la gestión estatal de la cultura y desarrollar un movimiento de masas original que trasforme radicalmente las subjetividades y el estatuto de lo artístico como parte de la totalidad material concreta, a la vez que absorba de las tradiciones, experiencias y prácticas preexistentes, los nutrientes necesarios para producir nuevos e inéditos fenómenos.

Estas han sido las incipientes y más profundas mordidas del primer caimán con barba. Gracias a ellas, este magazine se convirtió rápidamente en una de las más genuinas expresiones del pensamiento marxista antidogmático hegemónico en los iniciales años de la primera revolución socialista triunfante de América Latina.

\section{Bibliografía}

Acha, Omar. La nueva generación intelectual. Incitaciones y ensayos. Buenos Aires: Herramienta. 2008.

El caimán barbudo № 1 a 21 (1966-1968).

Díaz, Jesús. $<<$ Literatura revolucionaria $>>$. En Bohemia. Año 58, Nº 29 (1966): 26.

Díaz, Jesús. <<El último puente>>. En La Gaceta de Cuba Año V Nº 52 (1966): 4.

Estupiñán, Leandro. Lunes, un día de la Revolución Cubana. Bs As: Dunken. 2015.

Fornet, Ambrosio. $<<$ Quinquenio Gris: revisitando el término $>>$ en Ciclo La política cultural del periodo revolucionario: Memoria y Reflexión. La Habana: Criterios, 2007.

Fornet, Jorge. El 71. Anatomía de una crisis. La Habana: Letras Cubanas. 2013.

Gilman, Claudia. Entre la pluma y el fusil, debates y dilemas del intelectual revolucionario en América Latina. Buenos Aires: Siglo XXI. 2003.

Guanche, Juan. El continente de lo posible. Política y cultura en Cuba 19591968. La Habana: Ruth Editorial. 2008.

Guevara, Ernesto. Economía y Hombre nuevo. Lecturas para la reflexión 3. Buenos Aires: Ocean Sur. 2013.

Guerra Vilaboy, Sergio, Maldonado, Alejo. Historia de la Revolución Cubana. Txalaparta: Navarra. 2009. 
Hernández, Rafael, Rafael Hernández, $<<$ El año rojo. Política, sociedad y cultura en 1968>>. En Revista de Estudios Sociales N 33 (2009).

Kohan, Néstor. $<<$ Pensamiento Crítico y el debate sobre las Ciencias Sociales en el seno de la Revolución Cubana >>. En Crítica y teoría en el pensamiento social latinoamericano 389-435. CLACSO: Buenos Aires, 2006.

Martínez Pérez, Liliana. Los hijos de Saturno. Intelectuales y Revolución en Cuba. México: FLACSO. 2006.

Orta Ruiz, Jesús. $<<$ Respuesta fraternal a Jesús Díaz $>>$. En Bohemia, Año 58, N 31 (1966): 26-27.

Otero, Lisandro. Llover sobre mojado. Memorias de un intelectual cubano (19571997). México D.F.: Planeta. 2001.

Pogolotti, Graziella, ed. Polémicas culturales de los sesenta. La Habana: Letras Cubanas. 2006. 